\title{
RADIATION METHOD OF TEMPERATURE MEASURMENT IN THE ZONE OF LOCALIZED IMPACT LOADING
}

\section{I.B. CHERVIAKOV and D.V. LAPTEV}

Moscow Oil and Gas Institute, Department of Mechanical Engineering, Leninsky Prospect 65, Moscow 117917, USSR

\section{Abstract}

The radiation low-inertial method was developed for temperature measurment in the impact contact zone, using the pyrometer based on the non-cooled photoresistor and flexible fibreoptical cable. The pyrometer is attached to the "pneumatic gun" testing rig, which provides the collision of steel balls having the diameters up to $3 \times 10^{-3} \mathrm{~m}$ and other hard particles with the specimen surface at the velocities up to $300 \mathrm{~m} / \mathrm{sec}$. The impact angles vary continiously from 0 to $90^{\circ}$. This method allows to measure the temperatures in the impact zone of the materials with low and high electroconductivity and is advantageous in comparison with methods using thermocouples of different types. The experimental data are obtained for some technically pure metals and structural steels at various impact energies and angles. They show the experimental possibilities of this method and have their own scientific value.

\section{Introduction}

The hard particle-material surface interaction at collision is a non-stationary process and is characterized by high loading speed and low contact time. Obviously, the processes of heat emission at the contact area are as fast as the process of impact itself. It was found in /I/ that at the collision speed of two bodies $I 00 \mathrm{~m} / \mathrm{sec}$, the time of temperature rise in the loading zone is $10^{-4} \mathrm{sec}$ and less. At the same time cooling of the heated impact zone is much longer process than heating. Difference in time constants for these two pro- 
ceases is at least of one order and dependa upon the parameters of loading and thermal properties of the materials $/ 2,3 /$.

At high rates of the order of $\mathrm{IO}^{3}-\mathrm{IO}^{4} \mathrm{gec}^{-\mathrm{I}}$ the heat emission in the deformed volume of the material can be considered as adiabatic /4/. In this case all the thermal energy is concentrated in the given volume and in the moment of impact loading the energy of infrared radiation will correspond to the maximum emitted thermal energy.

\section{Method and materials}

The testing technique for temperature measurment in the zone of hard particle colligion with the surface of material using the pyrometer with fibre-optical cable was developed in Moscow 0 il and Gas Institute.

The functional block-diagram of the technique is given in Fig.I. The testing rig consists of compessed gas cylinder I, pressure reduction valve 2 , intermediate chamber 3 , standard pressure meter 4 , electromagnetic valve 5, loading device 6 for steel balls and the gun tube 7 .

Measurment of impact and rebound velocities $v_{0}$ and $v_{I}$ of the particle is mede in the following way. The particle crosses the light beam from the source (not show). The photodiod 8 generates the pulse triggering the clock II. At the collision of the particle with the specimen 9, the piezoceramic sensor, attached to the specimen, generates the pulse, stopping the clock II and triggering the clock I2. The next pulse, generated by the sensor Io at the impact of the rebounded particle, stops the clock I2. Knowing the distance between the positions of sensors 8-9 and 9-IO and the time shown by the clocks II and I2, we can calculate the velocities $v_{0}$ and $v_{I}$. The accuracy of velocity measurment is $\pm I \%$, the accuracy of the impact angle is $\pm I^{\circ}$.

This technique provides the following loading parameters: initial particles' velocities up to $-00 \mathrm{~m} / \mathrm{sec}$, impact angles $15-90^{\circ}$, particles diameters $\mathrm{I}-4 \mathrm{~mm}$.

The optical cable which transmits the infrared radiation from the interaction zone to the detector is made of Ge-As-Se material. The diameter of the optical core is 430 micrometers. For mechanical safety the cable is mounted in the steel tube and centered in it with plastic inserts. For decreasing energy losses due to reflections from the ends of the optical cable they were precisely polished. As a detector the non-cooled Se-Pb photoresistor of the type 
FR-64 was used. The technical characteristics of the detector allow to use it in the infrared region of the spectrum. The time constant of the detector is IO-I5 microseconds, allowing to analyze the thermal processes in the impact zone with the same time parameters.

The broad-band amplifier I5 is used for conditioning the signal, obtained from the sensor I4. The output pyrometric signal (voltage) Uout, corresponding to the monochrome radiation with a constant value of effective wavelength $\lambda$ eff, is directly related to the temperature of the radiating object. The oscilloscope I6 with memory is used for visualizing and recording the temperature pulsea generated by the pyrometer.

Before the measurments the instrument was calibrated against statically heated surfaces with known temperatures of the same materials used for dynamic tests by particles impacts. For modelling of the dynamic loading conditions the mechanical chopper was used, modulating the infrared flux from the heated body with the frequency of I $\mathrm{kHz}$. The preliminary experiments showed that the radiation modulation frequency does not affect the output parameters of the pyrometer up to the values of $0,5 \mathrm{MHz}$. In this way the pyrometer was calibrated in the dynamic conditions as close to the actual measurment process as possible.

The temperature measurment at the impact of the surface of tested material by the hard particle is performed in the following way. The input end of the optical cable I3 is positioned perpendicularly to the surface of the specimen 9 at the distance $I=d_{p}+2 m m\left(d_{p}-\right.$ diameter of the particle, $2 \mathrm{~mm}$ - the methodically chosen distance required for an unobstructed rebound of the particle) and is directed to the suggested point of ineraction. Then the shot is made from the "pneumatic gun" and the infrared radiation from the contact zone is recorded by the pyrometer into the memory of the oscilloscope I6. In order to eliminate the noise and interference triggering of the oscilloscope is synchronized with the moment of impact via the piezoceramic transducer 9. The calibration allows to convert the values of Uut into the temperature data.

The temperature measurments in the contact zone were performed using the materials shown in the Table $I$. 


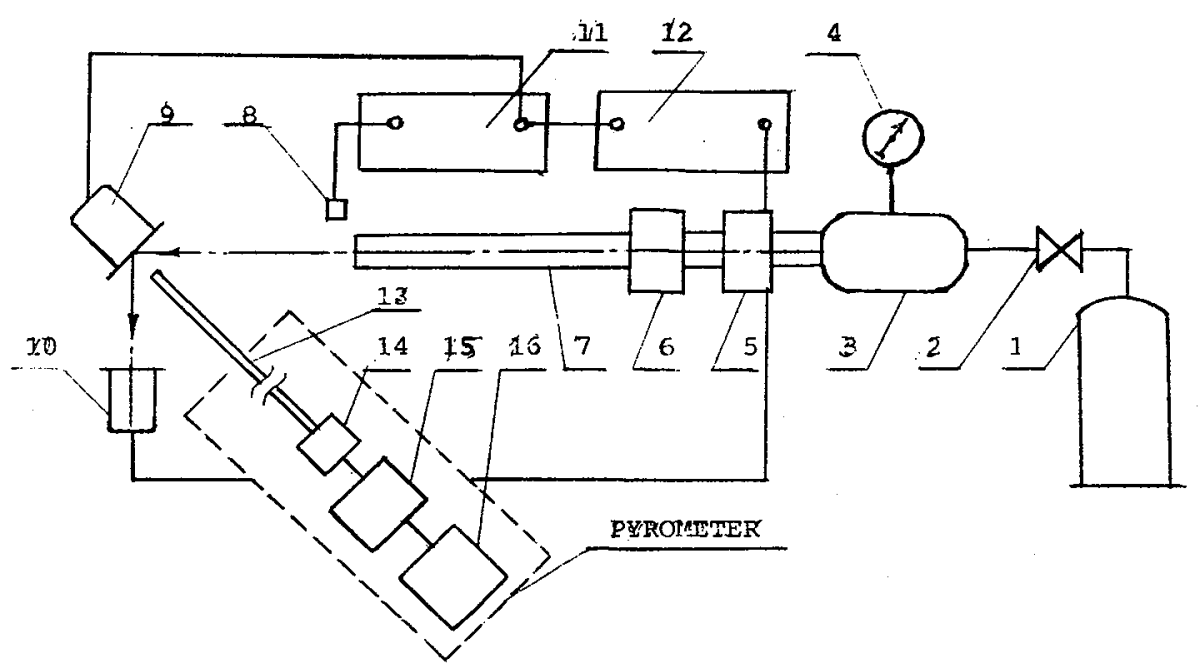

Fig.1 Block diagramm of the testing rig.

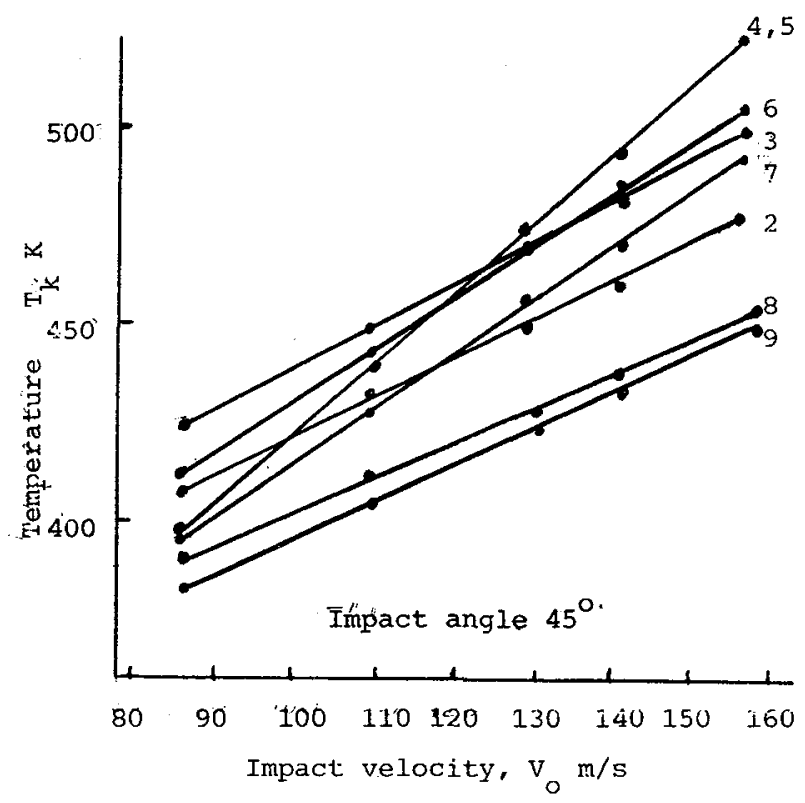

Fig. 2 Effect of particle velocity on the temperature in the collision zone

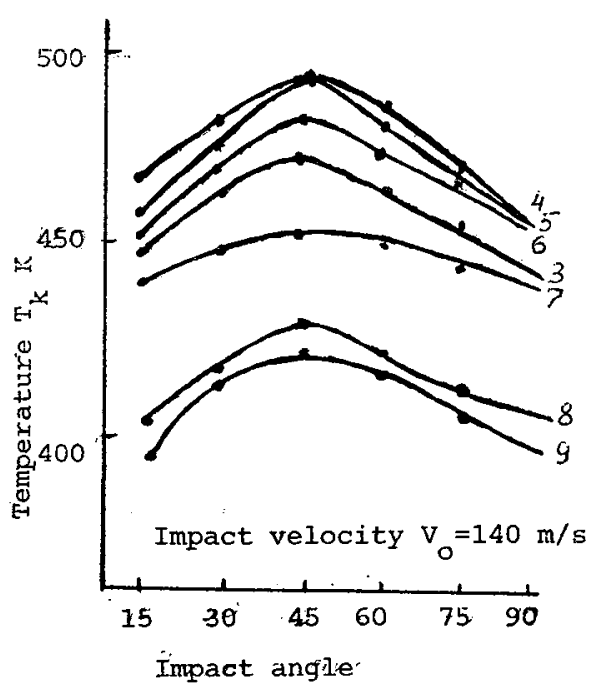

Fig.3 Effect of impact angle on the temperature in the collision zone. 
Table I

\begin{tabular}{|c|c|c|c|}
\hline No. & Material & Heat treatment & $\begin{array}{l}\text { Hardness } \\
\mathrm{HV}, \mathrm{MPa}\end{array}$ \\
\hline$I$ & $\mathrm{Cu}$ & - & I000 \\
\hline 2 & Fe Armco & - & I300 \\
\hline 3 & Steel 3 & - & $I 400$ \\
\hline 4 & Steel 45 & Normalizing & I900 \\
\hline 5 & Steel U8 & Normalizing & 2100 \\
\hline 6 & Steel 45 & $\begin{array}{l}\text { Quenched from } 850^{\circ} \mathrm{C} \\
\text { and tempered at } 500^{\circ} \mathrm{C}\end{array}$ & 3000 \\
\hline 7 & Steel u8 & $\begin{array}{l}\text { Quenched from } 850^{\circ} \mathrm{C} \\
\text { and tempered at } 470^{\circ} \mathrm{C}\end{array}$ & 3500 \\
\hline 8 & $\mathrm{Ti}$ & - & 3600 \\
\hline 9 & Mo & - & 4500 \\
\hline $\begin{array}{l}\text { Steel } \\
\text { balls }\end{array}$ & Steel $95 \mathrm{CrI8}$ & Quenched and tempered & $60-62 \mathrm{HRC}$ \\
\hline
\end{tabular}

\section{Results}

The experiments have shown that at higher initial velocities of impact the temperature in the collision zone increases (Fig. 2). This growth of temperature $\mathrm{T}_{\mathrm{k}}$ principally is determined by increased plastic deformation. The generation of high local heating in the deformed zone is caused mainly by the strain rate.

In case of high-speed deformation the heat has not sufficient time to dissipate, the process becomes adiabatic and leads to the temperature rise in the collision zone. The locality of temperature flash was proved by the following experiment. The optical cable of the pyrometer was focused on the zone adjacent to the supposed dimple. At collision the pyrometer in this case has not recorded any temperature pulse.

As it is seen from Fig. 2, the temperature $\mathrm{T}_{k}$ in the impact zone reaches the highest value of $530 \mathrm{~K}$.

It is interesting to observe the dependence of $\mathrm{T}_{k}$ upon the impact angle. It was shown that this dependence is characterized by the maximum at impact angles around $45^{\circ}$ (Fig. 3). This was observed for all tested materials and impact velocities used in these experiments, 
and can be explained by the simultaneous effects of the degree and the rate of plastic deformation upon the temperature $\mathbb{T}_{k}$. Decreasing of the impact angle $\alpha$ and consequently the degree of deformation leads to decreasing of the temperature $\mathrm{T}_{\mathrm{k}}$.

Significant, in our opinion, effect upon the temperature change in the collision zone is caused by the frictional events in the contact area. It was show /5,6/ that the friction coefficient at the collision of hard particle with the surface is not constant but has its maximum value in the region of sliding impact angles. So the temperature maximum $\mathrm{T}_{\mathrm{k}}$ around impact angles 40-50 is due to the additional heating of the contact zone by friction of the particle against the surface of tested material.

It was reported in $/ 7 /$ about the extreme adsorbtion by the material of initial kinetic energy of the particle in the range of impact angles $\alpha=25-45^{\circ}$ due to the sliding friction of the particle over the surface.

The experiments have shown that the size of the particles has very low effect on the temperature in the collizion zone. It is so, because the plastic deformation parameters, cesponsible for temperature growth, practically do not depend upon the size the particles. It is supported by the results of $/ 8 /$ where it was shown that the temperature in the contact zone is not sensitive to the particle size.

We did not found any explicit correlation between the temperature and material properties. Probably it is due to the temperature dependence upon the combination of strength, plasticity and thermal properties of the material. To distinguish the effects of each factor was not possible in this work.

\section{Conclusion}

The experimental technique has been developed, allowing to measure the temperature in the impact zone at the collision of hard particle with the surface of various materials. The pyrometer with non-cooled photoresistor and flexible fibre-optical cable is the part of this technique.

It was shown that the temperature in the contact zone rises continiously with rising impact velocity and achieves the value of $530 \mathrm{~K}$. The maximum of temperature was observed in the region of impact angles of $40-50^{\circ}$ and varies weakly with the size of the particles. 


\section{References}

I. Kononenko V.G. High-speed forming and fracture of metals (in Russian). Charkov, I980.

2. Babichev et al. Pribory i technika experimenta, I985, N 5, p. I97I98.

3. Vinogradov V.N. et al. Wear at impact (in Russian). Moscow, Mashinostroenie, I982.

4. Shock waves and high-speed deformation of metals (in Russian). Moscow, Metallurgia, I984.

5. Pateiuk G.M. Nauchnye trudy OMIIT, I965, v. 57, p. 67-76.

6. Styller E.E. Sbornik trudov VNIIPTUGLEMASH, Moscow, I975, issue 20, p. 75-8I.

7. Sorokin G.M. Vestnik mashinostroenia, I986, N 5, p. II-I5.

8. Kilchevsky N.A. The theory of solid bodies collision (im Russian). Kiev, Naukova Dumka, I969. 